Revue internationale P.M.E.

Économie et gestion de la petite et moyenne entreprise

\title{
De la démarche d'informatisation comme déterminant de la satisfaction informationnelle : une recherche exploratoire en contexte de P.M.E.
}

\section{Samir Blili}

Volume 2, numéro 1, 1989

URI : https://id.erudit.org/iderudit/1007906ar

DOI : https://doi.org/10.7202/1007906ar

Aller au sommaire du numéro

Éditeur(s)

Presses de l’Université du Québec

ISSN

0776-5436 (imprimé)

1918-9699 (numérique)

Découvrir la revue

Citer cet article

Blili, S. (1989). De la démarche d'informatisation comme déterminant de la satisfaction informationnelle : une recherche exploratoire en contexte de P.M.E. Revue internationale P.M.E., 2(1), 39-56.

https://doi.org/10.7202/1007906ar
Résumé de l'article

Une soixantaine de gestionnaires répartis dans trente PME manufacturières ont été invités à présenter leurs perceptions relatives au niveau de succès de leur système d'information organisationnel et au déroulement du projet informatique qui a conduit à sa mise en place. Ces données ont été traitées pour analyser la relation qui lie le processus d'informatisation avec la satisfaction informationnelle des utilisateurs. Les conclusions de cette étude tendent à renforcer la nécessité de développer une théorie spécifique de la gestion des systèmes d'information en contexte de PME. 


\title{
De la démarche d'informatisation comme déterminant de la satisfaction informationnelle : une recherche exploratoire en contexte de P.M.E.
}

\author{
Samir BLILI* \\ Université du Québec à Trois-Pivières
}

\begin{abstract}
RESUME
Une soixantaine de gestionnaires répartis dans trente PME manufacturières ont été invités à présenter leurs perceptions relatives au niveau de succès de leur système d'information organisationnel et au déroulement du projet informatique qui a conduit à sa mise en place. Ces données ont été traitées pour analyser la relation qui lie le processus d'informatisation avec la satisfaction informationnelle des utilisateurs. Les conclusions de cette étude tendent à renforcer la nécessité de développer une théorie spécifique de la gestion des systèmes d'information en contexte de PME.
\end{abstract}

\begin{abstract}
The relationship between the information systems development and implementation process and the success of such systems in the context of SMEs is analysed using empiral data obtained from 60 managers in computerized enterprises. The conclusions of this study tend to reinforce the specific character of the management of SMEs, and the opportunity to adapt theoritical frameworks and prescriptive models in regard to the implementation and management of computer-based information systems in the SMES context.
\end{abstract}

\section{RESUMEN}

La relacion entre el desarollo del sistema de informacionés, el proceso de su aplicacion y el éxeto de tales sistemas en el contexto de las PyME es analizada con datos obtenidos de 60 manageres de empresas dotadas de ordenadores. Las conclusiones de este estudio parecon reforzar el carácter particular del manager de las PyME, y la necessidad de utilizar en el contexto de PyME, de conceptos téoricos y modelos especialmente concebidas por la aplicacion y la organizacion de sistemas de informaciones a base de ordenadores.

* Samir Blili enseigne les systèmes d'information depuis plusieurs années. Il est professeur et coordonnateur des cours en systèmes à l'Université du Québec à Trois-Rivières et est un membre associé au GREPME. Son adresse est : GREPME, Université du Québec à Trois-Rivières, C.P. 500, Trois-Rivières, Québec, G9A 5H7. Première version reçue en mai 1989. 


\section{Introduction}

Préoccupation relativement récente de la gestion, la conception et l'implantation de systèmes d'information informatisés(SII)està la foisle résultat d'expériences pratiques, de recherches scientifiques et d'emprunts faits à d'autres disciplines. Le but à atteindre est de mettre au point un outil ayant pour fonction de servir le bon fonctionnementet d'assurer l'évolution des organisations. Or, depuis que les SII sont un domaine d'intérêt de l'administration, il s'est dit et écrit beaucoup de choses sur ce qu'ils sont, sur la façon de les concevoir et de les implanter, sur la façon dont ils performent et sur les pratiques requises pour leur gestion. Toutefois, il nous faut constater que peu de chercheurs se sont intéressés empiriquement à la question de l'implantation des SII dans le contexte spécifique des organisations de petite ou moyenne dimension. Certaines études ont cependant tenté de discriminer en fonction du critère de la taille, le niveau de succès ou d'insuccès de l'informatisation des systèmes de gestion des entreprises. À cet effet, Raymond (1984) a mis en évidence l'importance déterminante du contexte organisationnel particulier de la PME pour l'organisation, la gestion, l'implantation et l'utilisation des SII. Basée sur une approche contingencielle, son analyse a porté sur la relation qui lie divers facteurs avec le succès des SII.

Alors que la recherche empirique sur l'implantation des SII en contexte de PME présente certains manques, la documentation relative à l'informatisation des aides à la gestion des PME s'avère particulièrement abondante (Ahituv, 1981; Boland, 1978; Cadieux, 1989; Hooper, 1981; Laberge et Martin, 1983; Maloné, 1985; Pétro, 1983; Roberge, 1982; Taylor et Meinhardt, 1985; Weber et Tiemeyer, 1982). Cependant, en consultant cette documentation, on se rend compte que les recommandations qui y sont faites découlent de réflexions personnelles et ne reposent que très rarement sur des observations rigoureuses. Par contre, cette littérature témoigne del'ampleur du phénomène informatique; elle estaussi révélatrice des préoccupations que suscite l'insertion des SII au sein d'organisation de taille petite ou moyenne (Mayère, 1984). Ces constatations prouvent la nécessité d'élaborer et de valider un cadre normatif approprié à la conception et à l'implantation des SII dans la PME. Ce champ d'intérêt est sans doute l'un des plus traités dans la littérature professionnelle; les approches proposées allant du simple au complexe, l'objectif est de fournir aux intéressés un modele pratique des étapes à suivre pour franchir avec succès le seuil de l'informatisation ou pour changer de SII.

\section{Cadre conceptuel et hypothèses de recherche}

Notre recherche a pour but d'établir les bases conceptuelles relatives aux facteurs d'efficacité d'un processus de conception et d'implantation d'un système d'information informatisé dans un contexte de PME. Nous nous sommes donc 
intéressés à l'investigation empirique des relations existantes ou pouvant exister entre, d'une part, les caractéristiques du processus de conception et d'implantation (PCI) et, d'autre part, le succès du SII implanté. Pour le moment, les vérifications empiriques relatives à des modèles de conception et d'implantation de SII dans un contexte de PME ne paraissent être qu'à l'état d'embryons. Au départ, notre étude s'inscrit dans le courant de recherche de Powers (1971) et de Houle (1979) qui ont tenté de dégager les paramètres d'un modèle normatif de conception et d'implantation d'un SII dans une organisation.

Compte tenu des fondements théoriques et empiriques de la présente étude, un élément important consiste en l'élaboration d'un modèle cohérent du processus de conception et d'implantation d'un SII et de son application dans le contexte spécifique de la PME. En nous restreignant au contexte de la PME, nous postulons comme Fallery (1983) qu'on ne peut transposer systématiquement les théories des SII, essentiellement issues de l'observation de la grande organisation, dans la petite organisation parce que le contexte en est différent. Ce postulat a d'ailleurs amené Raymond (1984) à ébaucher un cadre conceptuel global des facteurs de succès d'un SII dans une PME, basé explicitement ou implicitement sur les relations présumées entre divers facteurs individuels, organisationnels ou techniques et divers indices de performance du système.

A un niveau plus global de la théorie des organisations, bon nombre d'auteurs soulèvent la nécessité de développer une théorie particulière à la PME (Gervais, 1978; Paulson et Stump, 1979; Welsch et White, 1981). Dans le même courant de pensée Welsh et White (1981) rappellent qu' «une P.M.E. n'est pas une petite grande entreprise», alors que Dandridge (1985), faisant allusion à la PME, soutient que «les enfants ne sont pas des petits adultes» et il poursuit en affirmant que «la P.M.E. a besoin de sa propre théorie». Aussi nous pensons qu'il est indispensable de donner de la PME une représentation spécifique. En ce sens, nous pouvons déduire que les facteurs d'efficacité d'un PCI validés en contexte de grande entreprise ne sont pas systématiquement applicables ou généralisables à la PME. Par contre, il est possible de se servir du corpus de connaissance sur les SII de la grande entreprise pour développer une théorie des SII de la PME. Cette logique est renforcée par Dandridge (1979) qui mentionne que la psychologie de l'enfant a fait bon usage de la psychologie qui vient de l'adulte; dans la même optique, il encourage les théoriciens à utiliser : «...les lunettes de la grande organisation, pour regarder la petite entreprise mais dans des perspectives qui lui sont propres». Or, il nous faut constater avec de nombreux théoriciens que la littérature empirique portant sur les systèmes d'information de la PME, à l'inverse de celle portant sur la grande entreprise, est peu foumie (Cheney, 1983; Raymond, 1984). C'est dans la même perspective que Blau, Heydebrand et Stauffer (1966) ont utilisé la simplicité structurelle de la PME afin de mieux saisir les relations entre certaines composantes de la grande entreprise. Nous pouvons donc déduire que les organisations peuvent être objets ou outils d'analyse. Pour ces derniers auteurs, la grande firme constituait l'objet de leur étude, en ce qui nous concerne, elle en est l'outil. 
Paradoxalement les conclusions de certainesétudes(Cheney, 1983) pourraient donner raison aux gestionnaires de PME qui en sont encore aux systèmes manuels de gestion de l'information. De fait, Raymond et Magnenat-Thalmann (1982), au terme de leur constat portant sur le degré de succès des systèmes d'information informatisés, concluent à l'absence de différences significatives entre PME informatisées et non informatisées en ce qui a trait à la satisfaction informationnelle de leur dirigeant. Ce constat d'échec apparent de l'informatisation de ces systèmes d'information nous incite à nous pencher sur les causes plausibles de cet insuccès. Ainsi, nous postulons que le PCI est effectivement un facteur de succès.

Nos objectifs sont : d'abord d'identifier les facteurs critiques de succès des projets informatiques en contexte de P.M.E., puis de mesurer le niveau de présences des facteurs ainsi retenus dans la réalité des processus d'informatisation, enfin, d'examiner la relation qui existe(rait) entre les facteurs d'efficacité et le succès du SI. Nos questions de recherches se présentent donc comme suit :

Quelles sont les conditions ou facteurs d'efficacité d'un processus de conception et d'implantation d'un SII dans une organisation de type PME?

Plus spécifiquement, nous nous sommes efforcés d'établir les paramètres d'un modèle normatif de cheminement efficace dans la conception et l'implantation d'un SII au sein d'une PME. La définition de ce processus «idéal» d'informatisation nous amènera à vérifier subséquemment la démarche effectivement suivie par les PME lors de la conception et de l'implantation de leur SII. D'où notre seconde question de recherche :

Par rapport au processus «idéal» (théorique) de conception et d'implantation d'un SII, y-a-t-il concordance avec le cheminement effectivement suivi par les PME qui utilisent ce type de système ?

Cette seconde question nous permettra de vérifier à quel point les processus adoptés par les PME convergent ou divergent de notre modèle théorique.

Finalement, dans le prolongement des travaux de Cheney (1983), de Kole (1983) et Raymond (1984), nous tenterons de vérifier l'impact des variables reliées à l'efficacité du processus de conception et d'implantation (PCI) sur le succès des SII en place dans les PME. A cet égard, notre troisième question de recherche est la suivante :

Y a-t-il une relation directe entre l'efficacité du processus de conception et d'implantation d'un SII dans une PME et le succès de ce système?

Cette dernière question suppose qu'on puisse définir et opérationnaliser le concept de succès d'un système d'information. Bien que peu d'efforts aient été faits en ce sens, il ressort qu' un certain consensus se dégage à ce niveau autour de la notion de satisfaction informationnelle, comme étant un indice approprié d'évaluation du succès (Hooper et Page, 1981). 
Processus d'identification des facteurs d'efficacité et leur présentation

Pour en arriver à identifier les facteurs d'efficacité, nous avons procédé à un examen de la documentation pertinente. Nousavons d'abord retenu les facteurs ayant un support empirique, et ensuite des facteurs non validés empiriquement mais pouvant plausiblement être considérés comme déterminants de l'efficacité du PCI. Ces derniers sont issus de recommandations ou d'exposés normatifs basés sur l'expérience ou le jugement des auteurs. Notre processus de sélection des facteurs d'efficacité est donc essentiellement fonction de l'appréciation de leur validité intrinsèque et de leur pertinence a priori. Pour en arriver à identifier les facteurs à retenir dans notre modèle initial de recherche, nous avons confronté l'ensemble des facteurs pré-sélectionnés à un examen de la documentation théorique et empirique portant sur la spécificité de la PME aux plans organisationnel, décisionnel, psychosociologique et fonctionnel.

Afin d'avoir une meilleure représentation des facteurs d'efficacité du PCI, nous avons choisi de les regrouper dans une version adaptée de la catégorisation de Houle (1979). Ainsi, ces facteurs sont répartis à travers quatre grandes catégories :

1) les facteurs reliés aux utilisateurs du SII;

2) les facteurs reliés au personnel préposé à la réalisation du SII;

3) les facteurs reliés à la conception du SII;

4) les facteurs reliés à la gestion du PCI.

Trois études importantes se veulent de base à notre cadre conceptuel. En effet, alors que l'étude de Raymond (1984) nous a permis d'identifier les facteurs de succès d'un SII en contexte de PME, les recherches de Powers (1971) et de Houle (1979), ainsi que les cadres conceptuels qui les sous-tendent, touchent à deux classes de variables faisant l'objet de notre étude: les caractéristiques du projet informatique et le succès du système implanté. Notons toutefois que les études de Powers et de Houle ont été entreprises dans un contexte de grande organisation. De plus, depuis le temps où ces deux études ont été réalisées, il s'est émis bon nombre de suggestions sur la façon de concevoir et d'implanter les SII. Le tableau 1 (p. 47 et 48) présente les 32 facteurs d'efficacité que nous avons finalement retenus. Notons aussi que les facteurs ayant trait aux utilisateurs du système fontréférence, soit aux caractéristiques de la participation des utilisateurs au projet, soit à des mesures critiques devant être prises pour favoriser l'utilisation. Les facteurs ayant trait au personnel préposé au PCIconcementessentiellement les attributs des personnes qui conçoiventet implantent le système, tandis que les facteurs ayant trait à la conception du système portent sur diverses étapes critiques de la réalisation du projet informatique, et que les facteurs ayant trait à la gestion du PCI sont constitués de caractéristiques critiques de la gestion du projet. L'objectif essentiel de notre étude est donc d'évaluer l'impact sur le succès du SII (variable dépendante) des caractéristiques critiques de l'efficacité du PCI (telles que spécifiées précédemment). La figure 1 (p. 44) présente notre modèle 
initial de recherche. Le succès du SII est mesuré en fonction de la satisfaction informationnelle de l'utilisateur (telle que perçue par les répondants). Ainsi, c'est une mesure d'efficacité du système implanté ou d'adéquation entre les attributs de l'information produite par le système (extraits) et son contexte d'utilisation (besoins et attentes des utilisateurs ou de l'organisation). La mesure de la satisfaction informationnelle est donc exprimée en fonction de la perception qu'ont l'utilisateur et le responsable informatique des attributs présumés du système (interface humainmachine, climat organisationnel à l'endroit du SII, soutien de la direction et potentiel opérationnel et décisionnel du système) et des attributs présumés de l'information qu'il produit (accessibilité, actualité, complétude, fiabilité,....). Chacun de ces attributs a été exprimé selon une échelle de type Likert à sept points (1 à 7). Le point 1 correspondant à un niveau très faible d'évaluation et le point 7 à un niveau très fort.

Fig. 1. Modele Initial de recherche sur les facteurs de succès du PCl en contexte de PME

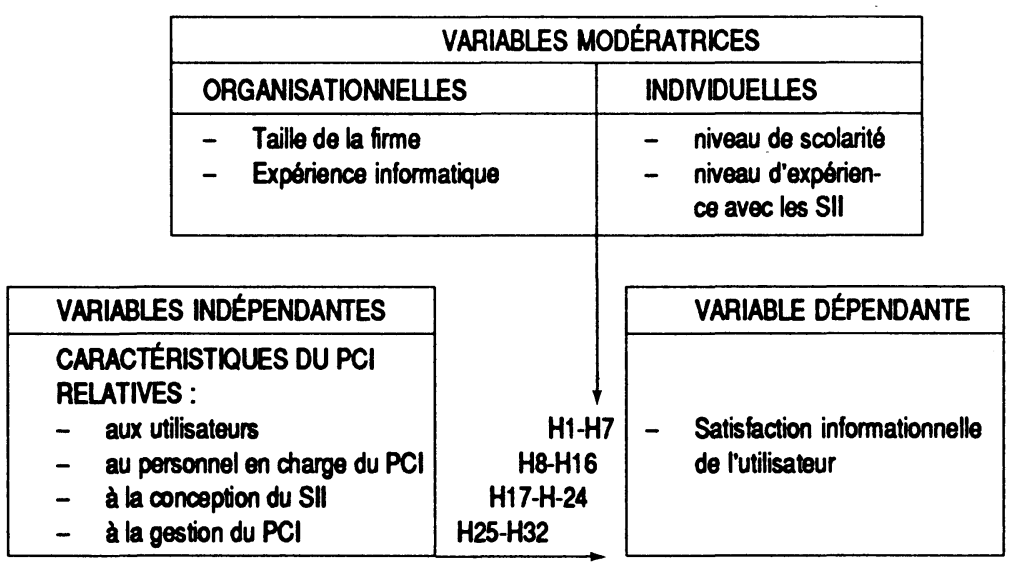

3 Hypothèses

Spécifiquement, nos hypothèses sont regroupéesen quatre grandes catégories:

1) les hypothèses mettant en relation les caractéristiques du PCI ayant trait à la participation des utilisateurs au processus de conception et d'implantation du SII avec la satisfaction informationnelle de l'utilisateur ( $\mathrm{H1}$ à $\mathrm{H7}$ );

2) les hypothèses mettant en relation les caractéristiques du PCI ayant trait au personnel affecté à la réalisation du projet avec la satisfaction informationnelle de l'utilisateur (H8 à H16); 
3) les hypothèses mettant en relation les caractéristiques du PCI ayant trait au processus de conception du SII avec la satisfaction informationnelle del'utilisateur (H17 a H24);

4) les hypothèses mettant en relation les caractéristiques du PCI ayant trait au processus de planification et de contrôle avec la satisfaction informationnelle de l'utilisateur (H25 à H32).Notre modèle comprend également des variables dites exogènes qui peuvent venir affecter la nature de la relation qui lie nos variables indépendantes avec les variables dépendantes. Pour nous, ces variables seront des variables modératrices en ce sens qu'elles sont considérées comme étant des sources potentielles d'interférence ou de biais. Ces variables sont réparties en deux classes (organisationnelles et individuelles) et sont au nombre de quatre.

\section{Méthodologie de l'étude empirique}

Notre échantillon se compose de 30 PME manufacturières de la province de Québec dont la taille varie de 11 à 300 employés. A l'intérieur de chacune de ces firmes, nous avons effectué des entrevues avec deux gestionnaires ( 60 au total). Dans chaque cas, nous avons recueilli les perceptions du principal responsable à l'interne du PCI et du principal utilisateur du système. La durée moyenne de ces entrevues est de $3 \mathrm{~h} 30$ environ. La mesure de la présence de facteurs d'efficacité et de satisfaction informationnelle a été faite au moyen d'un questionnaire comprenant des échelles métriques à sept points ( 1 à 7 ) ou selon une échelle dichotomique.

Le traitement des données et leur analyse a été faite à l'aide du progiciel SPSS (1975). Dans le but de répondre à notre seconde question de recherche, qui consistait à analyser l'impact de chacun des facteurs d'efficacité sur la satisfaction informationnelle de l'utilisateur, nous avons utilisé le coefficient de corrélation r de Pearson.

\section{$5 \quad$ Résultats relatifs à la présence des facteurs d'efficacité}

Il convient de se rappeler que le premier objectif empirique de l'étude est de vérifier si les responsables de la conception et de l'implantation des SII dans les PME tiennent compte des facteurs d'efficacité du PCI. Dans cette optique, nous vérifions dans quelle proportion les quatre catégories de facteurs se retrouvent dans la pratique des PME québécoises. Le tableau 1 (p. 47 et 48) présente les résultats sur le plan descriptif de la présence des 32 facteurs d'efficacité. 


\subsection{Présence des facteurs rellés aux utllisateurs du SII}

Les résultats du tableau 1 démontrent que les facteurs compris dans cette catégorie sont en majorité présents. Ainsi, en comparant les scores échantillonnaux moyens de chacun des sept facteurs, on peut conclure que les facteurs les plus respectés sont, dansl'ordre, la mise à la disposition desutilisateurs d'une documentation suffisante, la participation des utilisateurs à l'analyse des besoins, le sentiment de participation des utilisateurs. Dans la majorité des cas, on a implanté une structure formelle de formation des utilisateurs. Par contre, il semble que l'on ait insuffisamment fait participer les gestionnaires à la revue continuelle du projet et que, seulement dans $48 \%$ des cas, l'initiateur du projet ait été le président ou l'équivalent. Ce manque perçu au niveau de la participation des utilisateurs vient confirmer le caractère centralisé de la structure de la PME et le peu de cas qu'on y fait de la participation des subalternes au processus décisionnel (Mintzberg, 1979). On peut attribuer ces phénomènes à la mainmise et à l'omniprésence du propriétaire-dirigeant sur la gestion et les opérations(Blau et Heydebrand, 1966; Paulson et Stump, 1979). On n'accorde à l'entrepreneur qu'une faible propension à la délégation de pouvoir et à l'implication des employés dans la prise de décisions non programmées. En outre, on notera que dans la majorité des cas, le responsable du projet informatique a été le gestionnaire ayant charge de la fonction comptabilité/finance, ce qui renforce la spécificité fonctionnelle de la PME en regard des SII. La fonction systèmes d'information n'existant pas, les activités reliées à la gestion de l'information relèvent de ce dernier (Raymond, 1986).

\subsection{Présence des facteurs rellés au personnel préposé au PCI}

Les résultats démontrent que les facteurs ayant trait au personnel préposé au PCI sont majoritairement présents. En effet, il apparaît que dans respectivement $98 \%$ et $80 \%$ des cas, on a formellement désigné un responsable du PCI et on a fait appel à du personnel externe. La fréquence de ces deux facteurs laisse supposer que l'importance perçue du projet informatique fut relativement grande (désignation formelle d'un responsable) et que ces firmes sont effectivement dépourvues de personnel informatique (Turner, 1982). Tous les autres facteurs compris dans cette catégorie ont aussi été fortement considérés, exception faite de l'orientation du personnel informatique. Cette orientation est perçue comme étant insuffisamment axée sur les utilisateurs, ce qui confirme l'opportunité de procéder au développement des SII selon une méthodologie non traditionnelle (Doll et Mesbah, 1985), d'autant plus que les clivages entre informaticiens et gestionnaires sont vraisemblablement encore plus marqués dans un contexte de PME qu'ils peuvent l'être dans un contexte de grande organisation. Cependant, la provenance du personnel informatique (majoritairement externe) est un élément qui peut expliquer l'insatisfaction des utilisateurs vis-à-vis de l'impact de leurs besoins sur le PCI. En effet, compte tenu de l'importance des coûts de ce personnel externe, il est permis de croire que le niveau d'attente des utilisateurs vis-à-vis de celui-ci risque d'être plus élevé. 
Tab. 1. Statistiques descriptives des variables Indépendantes

\begin{tabular}{|c|c|c|c|c|c|}
\hline VARIABLES & $\begin{array}{l}\text { Moyeme } \\
(n-60)\end{array}$ & $\begin{array}{l}\text { Ecar- } \\
\text { ype }\end{array}$ & Mediane & $\begin{array}{l}\text { Moyenne } \\
\text { Utilisa- } \\
\text { lours } \\
(n=30)\end{array}$ & $\begin{array}{c}\text { Moyeme } \\
\text { Respon- } \\
\text { sebles } \\
(n=30)\end{array}$ \\
\hline \multicolumn{6}{|l|}{$\begin{array}{l}\text { Factours roliés aux } \\
\text { utilisatours du SII }\end{array}$} \\
\hline $\begin{array}{l}\text { 1. L'initiateur du projet est } \\
\text { le président" }\end{array}$ & 0,48 & & & 0,51 & 0,45 \\
\hline $\begin{array}{l}\text { 2. Participation des utilisateurs } \\
\text { à l'analyse des besoins }\end{array}$ & 4,10 & 2,10 & 4,00 & 4,10 & 4,20 \\
\hline $\begin{array}{l}\text { 3. Participation des utilisateurs } \\
\text { à l'approbation des spécifications }\end{array}$ & $4, \infty 0$ & 1,70 & 4,00 & 3,80 & 4,10 \\
\hline $\begin{array}{l}\text { 4. Participation des utilisateurs } \\
\text { à la revue du projet }\end{array}$ & 3,70 & 1,80 & 4,00 & 3,80 & 3,70 \\
\hline $\begin{array}{l}\text { 5. Sentiment de participation } \\
\text { des utilisateurs }\end{array}$ & $4, \infty$ & 2,30 & 4,00 & 3,40 & 4,60 \\
\hline $\begin{array}{l}\text { 6. Structure de formation } \\
\text { des utilisateurs" }\end{array}$ & 0,58 & & & 0,60 & 0,56 \\
\hline $\begin{array}{l}\text { 7. Documentation du SII } \\
\text { suffisante }\end{array}$ & 4,50 & 1,70 & 5,00 & 4,40 & 4,60 \\
\hline \multicolumn{6}{|l|}{$\begin{array}{l}\text { Factours reliés au } \\
\text { personnel proposé au PCl }\end{array}$} \\
\hline $\begin{array}{l}\text { 8. Désignation formelle d'un } \\
\text { responsable du PCr }\end{array}$ & 0,98 & & & 0,96 & $1, \infty$ \\
\hline $\begin{array}{l}\text { 9. Intervention de personnel } \\
\text { externe* }\end{array}$ & 0,80 & & & 0,80 & 0,80 \\
\hline $\begin{array}{l}\text { 10. Compétence tochnique du } \\
\text { personnel }\end{array}$ & 4,20 & 2,30 & 5,00 & 3,90 & 4,50 \\
\hline $\begin{array}{l}\text { 11. Compétence en gestion du } \\
\text { personnel exteme }\end{array}$ & 3,80 & 2,10 & 4,00 & 3,50 & 4,00 \\
\hline $\begin{array}{l}\text { 12. Expérience de la PME du } \\
\text { personnel externe }\end{array}$ & 4,10 & 2,30 & 5,00 & $4, \infty$ & 4,30 \\
\hline $\begin{array}{l}\text { 13. Expérience de la firme con- } \\
\text { sidérée du personnel du PCl }\end{array}$ & 4,90 & 1,80 & 5,50 & 4,70 & 5,10 \\
\hline $\begin{array}{l}\text { 14. Orientation de l'ensemble } \\
\text { du personnel du PCI }\end{array}$ & 3,30 & 2,10 & 4,00 & 3,30 & 3,40 \\
\hline $\begin{array}{l}\text { 15. Climat psychologique au } \\
\text { sein du projet }\end{array}$ & 4,60 & 1,90 & 5,00 & 4,40 & 4,70 \\
\hline $\begin{array}{l}\text { 16. Consensus relativement aux } \\
\text { objectifs du SII }\end{array}$ & 4,60 & 1,90 & 5,00 & 4,50 & 4,70 \\
\hline
\end{tabular}

- Échelle dichotomique : 1 : présence; 0 : absence 
Tab. 1 (suite) Statistiques descriptives des varlables indépendantes

\begin{tabular}{|c|c|c|c|c|c|}
\hline VARIABLES & $\begin{array}{l}\text { Moyenne } \\
(n=60)\end{array}$ & $\begin{array}{l}\text { Ecar- } \\
\text { type }\end{array}$ & Médiane & $\begin{array}{c}\text { Moyenne } \\
\text { Utilisa- } \\
\text { teurs } \\
(n=30)\end{array}$ & $\begin{array}{c}\text { Moyenne } \\
\text { Respon- } \\
\text { sebles } \\
(n=30)\end{array}$ \\
\hline \multicolumn{6}{|l|}{$\begin{array}{l}\text { Factours reliés à la } \\
\text { conception du SII }\end{array}$} \\
\hline $\begin{array}{l}\text { 17. Évaluation des procédés } \\
\text { ayant cours }\end{array}$ & 4,70 & 1,60 & $5, \infty$ & 4,50 & 4,80 \\
\hline $\begin{array}{l}\text { 18. Considération accordée aux } \\
\text { objectifs de la firme }\end{array}$ & 4,90 & 1,70 & 5,50 & 4,80 & 5,00 \\
\hline $\begin{array}{l}\text { 19. Analyse des besoins } \\
\text { informationnels" }\end{array}$ & 0,83 & & & 0,83 & 0,83 \\
\hline $\begin{array}{l}\text { 20. Formulation de priorités } \\
\text { relatives au SII" }\end{array}$ & 0,80 & & & 0,83 & 0,76 \\
\hline $\begin{array}{l}\text { 21. Rédaction d'un cahier de } \\
\text { charges* }\end{array}$ & 0,30 & & & 0,26 & 0,33 \\
\hline $\begin{array}{l}\text { 22. Réalisation d'un test } \\
\text { d'accoptation }\end{array}$ & 0,56 & & & 0,56 & 0,56 \\
\hline $\begin{array}{l}\text { 23. Réalisation d'une } \\
\text { conversion }\end{array}$ & 0,75 & & & 0,73 & 0,76 \\
\hline 24. Délais de réalisation du PCI & 17,40 & 9,20 & 17,0 & 17,90 & 17,00 \\
\hline \multicolumn{6}{|l|}{$\begin{array}{l}\text { Factours reliés al la } \\
\text { gestion du PCl }\end{array}$} \\
\hline $\begin{array}{l}\text { 25. Réalisation d'une étude } \\
\text { d'opportunité" }\end{array}$ & 30,00 & & & 30,00 & 30,00 \\
\hline $\begin{array}{l}\text { 26. Définition d'objectils } \\
\text { formels et réalistes }\end{array}$ & 5,30 & 1,30 & 5,50 & 4,70 & 5,80 \\
\hline $\begin{array}{l}\text { 27. Leadership de la haute } \\
\text { direction }\end{array}$ & 4,50 & 2,00 & $5, \infty$ & 4,40 & 4,70 \\
\hline $\begin{array}{l}\text { 28. Lancement d'un appel } \\
\text { d'offres' }\end{array}$ & 0,76 & & & 0,76 & 0,76 \\
\hline $\begin{array}{l}\text { 29. Réalisation d'une étude de } \\
\text { faisabilité }\end{array}$ & 0,26 & & & 0,30 & 0,23 \\
\hline $\begin{array}{l}\text { 30. Préparation d'un échéan- } \\
\text { cier d'implantation" }\end{array}$ & 0,45 & & & 0,40 & 0,50 \\
\hline $\begin{array}{l}\text { 31. Implantation d'une structure de } \\
\text { comptabilisation des activités du SII" }\end{array}$ & $0, \infty$ & & & 0,00 & $0, \infty$ \\
\hline $\begin{array}{l}\text { 32. Mise on oeuvre d'une structure } \\
\text { d'évaluation des objectifs du SII" }\end{array}$ & 0,00 & & & 0,00 & 0,00 \\
\hline
\end{tabular}

- Échelle dichotomique : 1 : présenco; 0 : absenco 


\subsection{Présence des facteurs rellés à la conception du système}

Les résultats du tableau 1 indiquent que ces facteurs sont présents dans une proportion de 6 sur 8 . Il semble que seuls les facteurs qui concernent la rédaction d'un cahier de charges et les délais de réalisation du PCI soient en général absents, puisque $30 \%$ seulement des firmes ont utilisé un cahier de charges et que le délai moyen de réalisation du PCI est de 17,4 mois. En rapprochant ce délai moyen au fait que 50\% des firmes ont un horizon de planification qui n'excède pas 12 mois, on peut déduire que ce délai est trop long. Le fait que seules neuf firmes aient utilisé un cahier de charges indique une fois encore le peu de complexité de la gestion de la PME ainsi que son manque de maturité en matière de projet informatique. En outre, la complexité relative du processus de rédaction de ce type de document à contenu fortement technique, ajoutée à l'insuffisance des ressources internes et aux coûts du personnel externe, sont autant d'éléments qui peuvent expliquerl'absence relative de ce facteur.

\subsection{Présence des facteurs rellés à la gestion du PCI}

L'interprétation des résultats présentés au tableau 1 nous permet de conclure que les facteurs compris dans cette catégorie sont majoritairement absents, soit dans une proportion de 5 sur 8. En effet, seule la définition d'objectifs formels et réalistes, le leadership exercé par la haute direction ainsi que le lancement d'un appel d'offres à plus d'un fournisseur semblent avoir été respectés. Notons que tel qu'attendu, aucune firme n'a procédé à l'implantation d'une structure de comptabilisation des activités du SII ou à la mise en place d'une structure d'évaluation du niveau d'atteinte des objectifs préétablis. Laprobabilité de retrouver ces deux facteursétaiteffectivement faible compte tenu de la spécificité fonctionnelle de la PME en regard des SII, de son mode de planification, de son niveau de formalisation ainsi que du style de gestion entrepreneuriale plutôt que technocratique (Gasse, 1986). Les mêmes raisons peuvent être invoquées pour expliquer l'absence relative de facteurs tels que la réalisation d'une étude d'opportunité ou de faisabilité et la préparation d'un échéancier d'implantation. Il est donc plausible de déduire que les caractéristiques organisationnelles, fonctionnelles et décisionnelles de la PME en regard des SII se réflètent sur le faible niveau de présence des facteurs d'efficacité ayant trait à la gestion du PCI. Par contre, la spécificité psycho-sociologique de ce type de firme, notamment l'omniprésence, le charisme et le pouvoir du propriétaire-dirigeant peut expliquer l'importance du leadership exercé par la haute direction durant le projet.pouvoir du propriétaire-dirigeant peut expliquer l'importance du leadership exercé par la haute direction durant le projet (Gasse 1986). 
Tab. 2. Corrélation des facteurs d'efficacité

avec la satisfaction informationnelle

\begin{tabular}{|c|c|c|}
\hline $\begin{array}{l}\text { VARIABLES } \\
\text { Factours rellis aux utillsatours }\end{array}$ & \multicolumn{2}{|c|}{$\begin{array}{l}r \text { avec contrôle des } \\
\text { variables modératrices }\end{array}$} \\
\hline $\begin{array}{l}\text { Facteure rellde aux utlllsateurs } \\
\text { Participation des utilisateurs a l'analyse des besoins } \\
\text { Documentation suffisante du SII } \\
\text { Participation des utilisateurs à l'approbation des spécifications } \\
\text { Structure de formation des utilisateurs } \\
\text { Participation des utilisateurs à la revue du projet } \\
\text { Sentiment de participation des utilisateurs } \\
\text { L'initiateur du projet est le propriétaire-dirigeant }\end{array}$ & $\begin{array}{r}0,547 \\
0,609 \\
0,487 \\
0,415 \\
0,416 \\
0,289 \\
-0,220\end{array}$ & $\begin{array}{l}\text { **** } \\
* * * \\
* * * \\
* * \\
*\end{array}$ \\
\hline $\begin{array}{l}\text { Facteurs rellde au personnel préposé au PCI } \\
\text { Consensus relativement aux objectifs du SII } \\
\text { Climat psychologique au sein du projet } \\
\text { Compétence technique du personnel externe } \\
\text { Orientation de l'ensemble du personnel du PCI } \\
\text { Compétence en gestion du personnel externe } \\
\text { Expérience de la PME du personnel externe } \\
\text { Expérience de la firme du personnel du PCI } \\
\text { Intervention de personnel externe } \\
\text { Désignation formelle d'un responsable du PCI }\end{array}$ & $\begin{array}{l}0,490 \\
0,692 \\
0,482 \\
0,481 \\
0,433 \\
0,489 \\
0,346 \\
0,253 \\
0,231\end{array}$ & "**" \\
\hline $\begin{array}{l}\text { Facteurs rellés la conceptlon du SII } \\
\text { Evaluation des procédés ayant cours } \\
\text { Considération accordée aux objectifs de la firme } \\
\text { Formulation de priorités relatives au SII } \\
\text { Délai de réalisation du PCI } \\
\text { Réalisation d'une conversion } \\
\text { Réalisation d'un test d'acceptation } \\
\text { Analyse des besoins informationnels } \\
\text { Rédaction dun cahier de charges }\end{array}$ & $\begin{array}{r}0,662 \\
0,585 \\
0,516 \\
-0,389 \\
0,174 \\
0,165 \\
0,153 \\
0,053\end{array}$ & **** \\
\hline $\begin{array}{l}\text { Facteurs rellés d la gestion du PCI } \\
\text { Définition d’objectifs formels et réalistes } \\
\text { Réalisation d'une étude de faisabilité } \\
\text { Etablissement d'un échéancier d'implantation } \\
\text { Lancement d'un appel dooffre } \\
\text { Leadership de la haute direction } \\
\text { Réalisation d'une étude d'opportunité } \\
\text { Implantation d'une structure de comptabilisation des activités } \\
\text { du SII (1) } \\
\text { Mise en oeuvre d'une structure d'évaluation } \\
\text { des objectifs du SII (1) }\end{array}$ & $\begin{array}{r}0,392 \\
0,316 \\
0,243 \\
-0,175 \\
-0,129 \\
-0,015\end{array}$ & ** \\
\hline
\end{tabular}

- $p<0,05$

** $p<0,01$

*** $p<0,001$

(1) tréquence $=0$. 


\section{Résultats relatifs aux hypothèses de recherche}

A ce stade, on se rappellera que notre second objectif empirique étant de valider un modèle de recherche qui associe certains facteurs d'efficacité du PCI à la satisfaction des utilisateurs d'un système d'information en contexte de PME. Ce modèle postule également l'existence de relations positives entre certaines caractéristiques organisationnelles ou individuelles de l'utilisation et la satisfaction informationnelle, cette dernière étant la mesure de succès retenue.

\subsection{Association des caractéristiques organisationnelles et Individuelles à la satisfaction}

Parmi les quatre variables modératrices de notre modèle de recherche, seule l'expérience des SII est associée significativement à une plus grande satisfaction informationnelle des utilisateurs $(r=0,241, p<0,05, n=60)$. A titre d'explication plausible à l'impact de cette variable sur le succès, mentionnons d'abord qu'il est possible que les utilisateurs les plus expérimentés peuvent être moins sujets à certaines formes de résistance au changement. Dans le même ordre d'idées, il est possible que des gestionnaires plusexpérimentés soientrelativement moins dépendants du personnel informatique, soient plus aptes à accéder au SII (utilisation) et soient plus à même de maximiser l'utilité de ce dernier. De plus, il est important de préciser que la satisfaction informationnelle est fonction entre autres des attentes et des besoins formulés par les gestionnaires à l'égard du système. De fait, nous sommes en droit de supposer que des gestionnaires plus expérimentés peuvent mieux anticiper la performance d'un SII a priori et ainsi éviter certaines désillusions. Par contre, il est possible que des gestionnaires peu ou non expérimentés puissent, compte tenu de l'engouement vis-à-vis de l'informatique, surestimer les capacités des SII. En outre, dans un contexte d'utilisation coutumière par opposition à une utilisation récente des SII, certaines difficultés techniques importantes, pouvant subvenir lors du projet, seront plus facilement contournées (Eason, 1977; Hedberg, 1975), ce qui favorise le déroulement du PCI et influence le succès du système.

\subsection{Association des facteurs d'efficacité reliés aux utilisateurs}

En ce qui concerne la première catégorie de facteurs, soit ceux reliés aux utilisateurs du système, l'examen du tableau 2 (p. 50) confirme l'importance de six des sept facteurs. Par ordre d'importance de leurs coefficients de corrélation, ces facteurs sont: la participation des utilisateurs à l'analyse des besoins (H2), la préparation d'une documentation suffisante relative au système (H7), la participation des utilisateurs à l'approbation des spécifications du système $(\mathrm{H} 3)$, la mise en oeuvre d'une structure de formation des utilisateurs (H6), la participation des utilisateurs à 
la revue continuelle du projet(H4)et le sentiment des utilisateurs d'avoir suffisamment participé au PCI (H5). Par contre, alors que ces six facteurs sont positivement associés à la satisfaction, le fait que l'initiateur du projet soit le président (H1) est négativementassociéà cette dernière; cette associationn'est toutefois pas significative.

Ces résultats tendent à confirmer les hypothèses mettant en relation les facteurs d'efficacité ayant trait aux utilisateurs du système avec la satisfaction informationnelle, ainsi que les résultats obtenus lors d'études précédentes. Rappelons que Lucas (1975) ainsi que Powers et Dickson (1973) ont trouvé que la formation des utilisateurs est positivement reliée au succès, que Houle (1979) et Raymond (1984) concluent que le niveau de compréhension du système est également relié au succès, que Doll et Ahmed (1985) ont trouvé l'existence de relations significatives entre la qualité de la documentation et le niveau de satisfaction vis-à-vis du système et que Boland (1978), contrairement à Powers et Dickson, a trouvé une relation positive entre la participation au PCI et la qualité du SII.

\subsection{Association des facteurs reliés au personnel préposé au PCI}

Revenant au tableau 2, on peut voir une corrélation significative entre huit des neuf facteurs qui ont trait au personnel préposé au PCI et la satisfaction informationnelle. Ces facteurs sont le climat psychologique au sein du projet (H15), le consensus relatif aux finalités du système (H16), la compétence technique du personnel externe (H10), le type d'orientation de l'ensemble du personnel du PCI (H13), la compétence en gestion (H11) ainsi que l'expérience de la PME (H12) du personnel externe et le niveau d'expérience de l'entreprise de l'ensemble du personnel préposé au PCI (H14). Ces résultats confirment donc ceux obtenus lors d'études précédentesetreflètentl'importance critique descaractéristiques du personnel informatique (Kole, 1983; Weber et Tiemeyer, 1981). Les résultats de Kaiser et Srinivasan (1982), relativement à l' impact sur le succès de l'orientation du personnel informatique, ainsi que ceux de Lucas (1975), relativement à la perception des utilisateurs à l'endroit du personnel informatique, semblent renforcés.

Les résultats relatifs à ces hypothèses, rapprochés de la catégorie de résultats qui les précède, contribuent à confirmer l'opportunité d'un style consultatif et adaptatif pour le personnel informatique intervenant dans la PME. A ces attributs importants s'ajoutent une orientation axée vers les utilisateurs, des habiletés à la communication, une expérience de l'informatique appréciable et une expérience de la gestion des PME qui soit suffisante.

\subsection{Association des facteurs reliés à la conception du système}

Encore au tableau 2, on peut voir que quatre facteurs parmi les huit ayant trait à la conception du système semblent significativement associés à la satisfaction informationnelle. Cesquatre facteurs d'efficacitéconcernentl'évaluation des procédés 
ayant cours (H17), la considération accordée aux objectifs de la firme (H18), la formulation de priorités relatives au SII (H20) et les délais de réalisation du PCI (H24). Concernant ces délais, mentionnons que contrairement à ce qui avait été prévu, les résultats tendent à démontrer que la relation qui les associe à la satisfaction est négative, ce qui contredit les conclusions de Houle (1979) à ce propos. Par contre, ces résultats renforcent les conclusions de ce dernier quant à l'importance accordée aux objectifs de la firme. Les hypothèses mettant en relation la satisfaction informationnelle à l'analyse des besoins informationnels (H13), à la rédaction d'un cahier de charges (H21), à la réalisation d'un test d'acceptation (H22) et à la réalisation d'une conversion (H23) ne sont pas confirmées. Ces résultats sont donc plus mitigés.

\subsection{Association des facteurs reliés à la gestion du PCI}

La demière section du tableau 2 concerne les résultats relatifs aux hypothèses qui associent les facteurs d'efficacité ayant trait à la gestion du PCI à la satisfaction informationnelle. Parmi les huit hypothèses comprises dans cette catégorie, seulement trois ont obtenu des résultats significatifs. Ces hypothèses concernent l'association de la satisfaction des utilisateurs avec la définition d'objectifs formels et réalistes (H26), la réalisation d'une étude de faisabilité (H23) etl'établissement d'unéchéancier d'implantation (H30). Notons que Houle a, lui aussi, trouvé que la définition d'objectifs relatifs au système est reliée à la satisfaction informationnelle. Contrairement à ce qui avait été prévu, les facteurs qui ont trait au leadership de la haute direction (H27) et au lancement d'un appel d'offres(H28) semblentnégativement reliés à la satisfaction. En outre, la réalisation d'une étude d'opportunité (H25) ne semble pas être un facteur affectant la satisfaction de l'utilisateur.

En ce qui concerne les hypothèses touchant à l'implantation d'une structure de comptabilisation (H31) et à la mise en oeuvre d'une structure d'évaluation du niveau d'atteinte des objectifs prétablis (H32), les résultats obtenus ne permettent pas de se prononcer, étant donné l'absence de ces facteurs dans notre échantillon. Les résultats portant sur le dernier groupe d'hypothèses laissent croire que les facteurs ayant trait à la gestion du PCI ont peu d'impact sur la satisfaction des utilisateurs, la majorité des hypothèses portant sur cette catégorie de facteurs n'étant pas confirmée. La première explication de cette absence apparente d'impact réside possiblement dans le fait que cette catégorie de facteurs nécessite un certain niveau de compétence en gestion qui semble peu courant au sein des PME. De plus, il n'a été que très rarement possible d'analyser en profondeur la teneur et les caractéristiques de ces étapes du projet en raison du fait que la documentation afférente au projet $n$ 'était pas accessible ou n'existait pas. Finalement, la majorité de ces facteurs n'ont pas été respectés en raison de la spécificité de la PME en matière de gestion et du contraste existant entre, d'une part l'empirisme des modes de gestion ayant cours dans la PME et, d'autre part, la systématisation requise pour les méthodologies d'implantation d'un SII. 


\section{$7 \quad$ Limites et contributions de la recherche}

La portée des conclusions de la présente étude est restreinte par un certain nombre de limites conceptuelles et méthodologiques qu'il convient de considérer. Au plan conceptuel, notre modèle de rechenche omet divers facteurs organisationnels, individuels et technologiques de l'utilisation des SII qui peuvent de façon tout à fait plausible affecter chacune de nos variables et leur relation. Au plan méthodologique les limites principales de cette recherche se situent au niveau de la nature et de la taille de notre échantillon, ce qui affecte sensiblement le pouvoir de généralisation des résultats. Les contributions de cette recherche sont de deux types : celles ayant trait à ses apports théoriques ou conceptuels, et celles qui touchent à son utilité sociale ou pratique. Au plan théorique, cette recherche a tout d'abord permis d'identifier un ensemble de trente-deux facteurs d'efficacité du PCI dans une PME. De plus, la confrontation de ces facteurs à un examen approfondi de la spécificité de la PME a permis de contribuer au développement d'un cadre conceptuel relatifà la particularité de la PME en regard des systèmes d'information. Au niveau de son utilité sociale ou pratique, cette recherche peut être utilisée par les concepteurs, analystes en systèmes, gestionnaires ou consultants oeuvrant pour le compte ou au sein d'une PME, comme cadre normatif ou guide pour la réalisation de projets informatiques orientés en fonction d'une meilleure compréhension des gestionnaires.

\section{Conclusion}

Conformémentà nos objectifs, nous avons suivi un certain nombre d'étapes dont les résultats confirment la spécificité de la PME en regard des systèmes d'information. La première étape de cette recherche visait à «positionner» l'étude dans le contexte théorique et empirique qui la sous-tend et à synthétiser, àl'aide d'un examen de la documentation pertinente, les facteurs d'efficacité du PCI en contexte de PME. Au terme de la confrontation de ces facteurs à un examen de la spécificité de la PME, il a été possible de regrouper ces derniers en quatre catégories, soit les facteurs ayant trait aux utilisateurs, au personnel en charge du PCI, à la conception du système et à la gestion du PCI. Cette démarche nous a permis de renforcer l'importance du contexte organisationnel en regard des projets informatiques, et ainsi, de dégager les parametres de la spécificité de la PME en regard du PCI.

A la lumière des données recueillies, nous sommes en mesure de conclure qu'effectivement, les PME s'inspirent dans leur démarche informatique des recommandations comprises dans la littérature. A ce titre, il apparaît que sont présents, par ordre de leur fréquence respective : les facteurs ayant trait au personnel 
préposé au PCI; les facteurs ayant trait aux utilisateurs; les facteurs ayant trait à la conception du système. Par contre, les facteurs ayant trait à la gestion du PCI sont majoritairement peu respectés. Ce fait peutêtre atribuable à la spécificité managériale de la PME.

Le second groupe de résultats, qui correspond à l'impact de ces facteurs sur le succès du SII, présente un profil semblable au précédent. Ainsi, il a été possible de relier les trois premières catégories de facteurs au succès du SII, alors que les facteurs portant sur la gestion du PCI ne semblent pas affecter le succès. Dans une optique d'élaboration d'un modèle normatif du processus de conception et d'implantation d'un SII et de son application dans le contexte spécifique de la PME, il semble que les facteurs d'efficacité ayant trait aux utilisateurs, au personnel informatique ainsi qu'à la conception du système soient effectivement à promouvoir. Par contre, il semble que les facteurs d'efficacité ayant trait à la gestion du PCI ne soient pas déterminants actuellement, d'où la nécessité de développer des méthodes et techniques de gestion du PCI plus adaptées à la réalité de la PME.

\section{BIBLIOGRAPHIE}

Ahituv, N., (1981), «Techniques of selecting computers for small business». Cahier de recherche no 56, Faculty of Calgary, Alberta, $15 \mathrm{p}$.

Auvolat, M., (1984), «800 000 Entreprises artisanales entre le micro et le réseau». Économie et humanisme, no 275, janvier-février, pp. 23-36.

Blau, P. M., Heydebrand, W. V. et Stauffer, R. E., (1966), «The structure of small bureaucracies». American Sociological Review, vol. 31, avril, pp. 179-191.

Boland, R., (1978), «The process and product of systems design». Management Science, vol. $24, n^{\circ}$ 9, mai, pp.

Cadieux, J. Y., (1979), «The application of mini computers to small business». 24 congrès annuel de International concil for small business, communication $n^{\circ} 52$, Québec, $10 \mathrm{p}$.

Cheney, P. H., (1983), «Getting the most of your first computer system». American Journal of Small Business, vol. VII, $\mathrm{n}^{\circ} 4$, avril-juin, pp. 50-60.

Dandridge, T. C., (1979), «Children are not little grown-ups : Small business needs its own organizational theory *. Journal of Small Business Management, avril, pp. 53-57.

Doll, W. J.et Mesbah, A.U., (1985), «Documenting information systems for management : A key to maintaining user satisfaction*. Information and Management, vol. 8, pp. 221-226.

Eason, K., (1977), kHuman Relationships and User Involvement in Systems Designx, Computer management, May, pp. 10-12.

Fallery, B., (1983), «Un système d'information pour les PME». Revue française de gestiom, novembre-décembre, pp. 70-76.

Gasse, Y., (1986), «The Processing of Information in Small Business and the Entrepreneur as Information Processor $*$ Communication à la $40^{\circ}$ rencontre annuelle de the Academy of management, Detroit, Michigan, août, 13 p.

Gervais, M., (1978), «Pour une théorie de l'organisation de la PME». Revue française de gestion, mars-avril, pp. 37-49.

Hedberg, B., (1975), «Computer Systems to Support Industrial Democracy», dans Mumford and Sakman (éd), Human choice and computers, North Holland. 
Hooper, P. et Page, J., (1981), «The promise of small business systems». Journal of Systems Management, vol. 32, $n^{\circ} 3$, septembre, pp. 17-27.

Houle, Y. (1979), «Les facteurs de succès d'un système d'information et leur relation avec la satisfaction informationnelle». Thèse de doctorat non publiée, Université Laval.

Ives, B., Olson, M. H. et Baroudi, J., (1983), «The measurement of user information satisfaction*. Communicationf à $A C M$, vol. $26, \mathrm{n}^{\circ} 10$, octobre, pp. 785-793.

Kaiser, K. et Srinivasan, A., (1982), «User-analyst differences : An empirical investigation of attitudes related to systems development». Academy of Management Journal, vol. $25, \mathrm{n}^{\circ}$ 3, pp. 630-646.

Kole, M. A., (1983), «Going outside for MIS Implementation» Information and Management, vol. 6, pp. 261-268.

Laberge, F. et Martin, L., (1983), «Comment vaincre la peur de l'ordinateurs. Le magazine des Affaires, vol. 6, $n^{\circ} 2$, mars, pp. $28-48$.

Lucas, H. C., (1975), Why Information Systems Fail, New-York, Columbia University Press.

Malone, S.C., (1985), «Computerizing small business information systems», Journal of Small business Management, vol. 23, $\mathrm{n}^{\circ} 2$, avril, pp. 10-16.

Mayere, A., (1984), «Trois millions de petites entreprises face à l'informatique». Économie et humanisme, $\mathrm{n}^{\circ} 275$, janvier-février, pp. 6-8.

Mintzberg, H., (1979), The Structuring of Organizations, Englewood CLiffs, New-Jersey, Prentice-Hall.

Nie, N. H. et al., (1975), A Statistical Package for the Social Sciences. New-York, McGrawHill.

Paulson, S. K. et Stump, A. L., (1979), «Small business and the theory of small bureaucracies». American Journal of Small Business, vol. 3, $\mathrm{n}^{\circ} 3$, janvier, pp. 34-47.

Petro, L. W., (1983), «Mini-computer systems for small business $\$$.Journal of Small business Management, juillet, pp. 1-6.

Powers, R. F., (1971), «An empirical investigation of selected hypotheses related to the success of management information systems projectsw. Thèse de doctorat, University of Minnesota, Minnesota.

Powers, R. et Dickson, G., (1973), «MIS project management : Myths, opinions and realities». California Management Review, vol. 15, $\mathrm{n}^{\circ} 3$, printemps, pp. 147-156.

Raymond, L. et Magnenat-Thalman, N., (1982), «Information systems in small business : Are they used in managerial decisions ?» American Journal of Small Business, vol. 6, $n^{\circ} 4$, avril-juin, pp. 20-26.

Raymond, L., (1984), «Uneétude empirique des facteurs de succès d'un système d'information en contexte de PME». Thèse de doctorat non publiée, Ecole des Hautes Études Commerciales, novembre.

Raymond, L., (1986), «The organizational validity of information systems in the context of small business». "Proceeding" du 14 e colloque annuel de ASAC, vol. 7, section 4, pp. 1-12.

Roberge, Y., (1982), «La puissance irrésistible des micro-ordinateurs». Le magazine des affaires, vol. $5, n^{\circ} 3$, avril, pp. 34-54.

Taylor, R. E. et Meinhardt, D. J., (1985), «Defining computer information needs for small business : A delphi method». Journal of Small Business Management, avril, pp. 3-9.

Tumer, J.A., (1982), «Firm size, performance and computer use», «Proceeding» de la troisième conférence annuelle en systèmes d'information, Ann Arbor, University of Michigan, Michigan, décembre, pp. 109-120.

Weber, H., et Tiemeyer, E., (1981), «Teaching information systems to small business management». Information and Management, vol. 4, pp. 297-303.

Welsh, J. A. et White, J. F., (1981), «A small business is not a little big business». Harvard Business Review, vol. 59, $\mathrm{n}^{\circ}$ 4, juillet-août, pp. 18-32. 